\title{
Extended scatterers model for fast sonar signal simulation
}

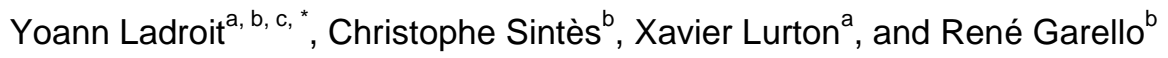 \\ ${ }^{a}$ Ifremer/IMN/NSE/AS, BP70, 29280, Plouzané, France \\ ${ }^{b}$ Telecom Bretagne/ITI, Technopôle Brest-Iroise, CS 83818, 29238, Brest Cedex 3, France \\ ${ }^{c}$ DGA/MRIS, 7 rue des Mathurins, 92221, Bagneux, France \\ *: Corresponding author : Yoann Ladroit, email address : yoann.ladroit@ifremer.fr
}

\begin{abstract}
:
Sonar signal simulation is an important area of research for industrials and scientific of the domain. Indeed, it can be used in many ways by sonar manufacturers, both for designing a system or in order to explain physical phenomenon on data. Therefore, by minimizing the expensive testing time at sea, a well designed simulator can be a very interesting financial asset.

Traditional sonar signal simulation also contains problems to be dealt with. It is often necessary to find a balance between the computational power consumption, the needed accuracy of the physical phenomenon description and the scale at which simulated signals are relevant.

In our simulation model we propose a solution based on a very dense grid (resolution of about the concerned wavelength) of extended scatterers spread on the seafloor. It is designed to describe efficiently the backscattering strength and the phase response of the seafloor for every type of sonar.
\end{abstract}

Keywords: Apertures ; Backscatter ; Computational modeling ; Electron tubes ; Mathematical model ; Sonar equipment

\section{CONTEXT AND INTRODUCTION}

A robust and efficient sonar signal simulator can be a useful tool for sonar manufacturers,and research laboratories Indeed, a well-built simulation code can be used as a designing or a testing tool for all these community. It can be used for developing new types of acoustical systems, for improving the performances of an existing one, or even for understanding phenomena observed on data already acquired. All those complementary aspects make sonar simulation a powerful mean of reducing the cost linked to the development and the use of sonar systems.

A great number of existing sonar simulators have already been made available by the scientific community [9]. The different ways of conceiving a model are directly linked to the computational power available and the accuracy needed in the physical phenomenon description. They are usually based on either ray tracing or tube tracing. 
Both approaches have their drawbacks and advantages. The solution proposed here is an intermediate solution to create a complete signal computation from an modelled seafloor and for a large set of sensors. This solution makes it possible to describe correctly both the backscattered energy of the seafloor and its phase characteristics. Therefore, it is adapted for all sorts of array processing issues.

At first, we will have a discussion on existing sonar simulations, and explain how the proposed model of extended scatterers differs from what has been implemented before. This model will then be fully detailed to justify its relevance. In the second part, we will validate the generated signals on existing theoretical models. Then, a few examples of the proposed algorithm will be presented. Finally, we will discuss the perspectives of improvement in the modelling and its limitations.

\section{GENERAL MODEL PRINCIPLES}

The model proposed here has been created in order to have a fast portable sonar simulation convenient to a great range of sensors, from the simple hydrophone to the multibeam echosounder.

It was designed to be able to describe correctly the phase response of the seafloor (as a ray tracing simulation [1]) and the backscattered energy (as a tube tracing simulation [2] [3]) in order to simulate a large range of acoustic sensors.

\section{A. Discussion on ray and tube tracing}

In a ray tracing model [6], the backscattered energy measured, issued from an aggregation method, depends on the density of rays intersecting a given area. Therefore, it supposes that the density is high enough, regardless of the geometry of the scene, to describe correctly the response of the seafloor. If not, then it will not be able to give a correct evaluation of the signal energy. This can be addressed by increasing greatly the ray density, but it results in a heavy computational power.

This aggregation problem can be solved by the method of tube tracing. The tubes are delimited by four rays, intersecting a certain area of the seafloor. From the area intersected, it is possible to get a good evaluation of the backscattered energy. Therefore it is possible to use this type of simulation to generate side-scan sonar data [2]. But this technique does not allow the simulation to render efficiently the statistical property of the seafloor. This is a important limitation as this aspect is needed to simulate array processing such as interferometry or beamforming, and physical phenomena such as baseline decorrelation or footprint shift [4]. Moreover, the intersection computation is very intensive in term of computational power and makes the whole process very demanding.

The extended scatterers model presented hereafter gives an alternative solution to these models in order to create a 
complete seafloor generated signal describing correctly energy and signal statistics property at the same time.

\section{B. Extended scatterers model}

1) Seafloor modelisation and extended scatterers density: The extended scatterers model is defined as follows. A regular rectangular grid $(x, y)$ with a resolution close to $\lambda$ (wavelength of the simulated sonar) is created. Each node of the grid is associated with an elevation $z$, describing the global geometry of the seafloor. Each small delimited surface represents an extended scatterer $e_{i}$. Each scatterer $(i)$ has therefore a fixed identity composed of:

- its center position $(x, y, z)$

- its area $S_{i}$

- its area normal $\overrightarrow{n_{i}}$

- a Rayleigh distributed amplitude $a_{i}$ with $\mathbb{E}\left[a_{i}\right]=B S_{0}$, $B S_{0}$ being the surface backscattering strength of the seafloor at $0^{\circ}$ incidence angle (in $\mathrm{dB}$ )

- a uniformly distributed phase response $\phi$ on $[-\pi, \pi]$

Considering an elementary surface with each associated scatterer provides a correct evaluation of the backscattered energy.

The crucial parameter in this modelling is the grid resolution. Indeed, in order to describe correctly the interferometric phenomenon, it is necessary to have enough extended scatterers inside the instantaneous insonified area [7]. This area will be delimited as shown in Figure 1.

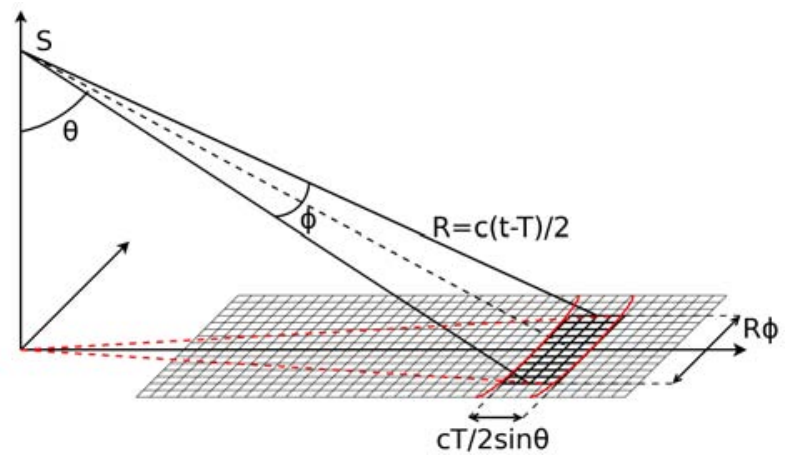

Figure 1. Insonified area according to incidence.

As explained in [5], the insonified area $A$ depends on the incidence angle of the pulse. At grazing incidence it can be approximated as $A \approx \Phi R \frac{c T}{2 \sin \theta}$.

Obviously it is better to have as much scatterers as possible, but using this model, it was found that the generated signals describe correctly the effects of footprint shift and baseline decorrelation (see III-A and [4]) with about a hundred scatterers in $A$. For most practical purpose, using a grid with a resolution of $\lambda$ will be appropriate.

The seafloor model remains then fixed all along the simulation, so that we are able to run several lines over it with different sensor configurations, viewing angles, or survey speeds. For each simulated position of the survey vessel, a part of the simulated seafloor will be selected according to the aperture of the sensors.

2) Array simulation: In order to reduce the computational power needed, the transmission is only characterized by its resultant spatial directivity. For instance, for a MBES, we will consider that the pulse is created by one hydrophone with a very thin along-track aperture (about $1^{\circ}$ ) and very wide acrosstrack aperture (about $140^{\circ}$ ). This could easily be changed to simulate the complete transmitting array, but would increase the simulation time by the number of hydrophones present in the transmitting array.

The receiving part of the simulation is completely simulated. Each hydrophone is considered separately. This offers the possibility to test several baselines, elementary directivities and array shadings.

3) Complex received signal computation: The pulse is produced and propagated from the center of the transmitting antenna to the center of each extended scatterer, which reflects back an energy flux. The value of this flux depends on the scatterer area, the angle $\theta_{s}$ between the normal to its surface and the propagation vector $\vec{I}$, and its amplitude (see Figure 2). Simultaneously, the phase of the backscattered wave is also shifted by the phase attributed to the scatterer.

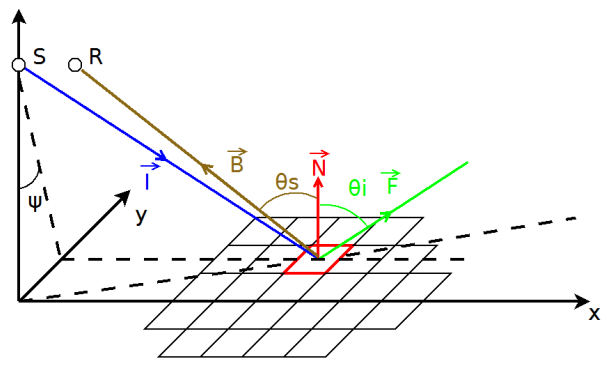

Figure 2. Reflection (green) and backscattering of the wave (brown) on the extended scaterrer.

In first order approximation, to compute the backscattered energy we assume that: $\theta_{i} \approx \theta_{s} \approx \theta$ since the distance between the source and the receiver is very small regarding the water depth. Therefore, for each extended scatterer, the backscattered wave takes this form:

$$
c_{i}(t)=s_{i}(t) e^{j w_{p} t} D_{\text {along }}(\psi) S_{i} a_{i} e^{j \phi_{i}} f(\theta)
$$

Where $s_{i}(t) e^{j w_{p} t}$ is the incoming wave at the instant $t$, and $D_{\text {along }}(\psi)$ is the along-track directivity of the antenna depending on the along-track angle $\psi$.

$f(\theta)$ is the function describing the strength of the backscattered signal according to the angle $\theta$. In first approximation, we can use Lambert's Law where $f(\theta)=\cos ^{2}(\theta)$. $a_{i}$ is Rayleigh distributed with $\mathbb{E}\left[a_{i}\right]=B S_{0}$, which is the backscattering strength per square meter of the surface. 
The forward scattered (to be considered for specular returns) wave is considered under this form :

$$
c_{i}(t)=s_{i}(t) e^{j w_{p} t} S_{i} a_{i}
$$

We assume that we have a perfect mirror reflection with no phase shift of the incident wave.

Then, a propagation loss is applied to the backscattered and forward-scattered wave. It is important to emphasize that throughout all the process the sonar equation is respected in order to be able to have a correct energy summation.

For a given ping and a given receiving hydrophone we identify which scatterers are insonified and are therefore contributing to the returning signal at a given time. Those contributions are summed to create the complex signal received by the hydrophone. Finally, the signal is completed with a Gaussian circular additive noise.

At the moment, this instantaneous insonified area computation is geometrically done under the assumption of a homogeneous sound velocity profile in the water column, and therefore with straight ray processing. The extension to a more realistic sound velocity profile will not change the model but only the geometry computation of the ray tracing.

\section{GENERATED SIGNAL VALIDATIONS}

This simulation was built with two main goals:

- producing signals describing the main statistical effects due to the interaction of the acoustics waves with the seafloor;

- computing properly the backscattered energy

In order to validate the good behaviour of the generated signals in those aspects, we went through a series of tests. Statistical behaviour is compared to theoretical expectations and angular backscatter response to usual MBES measurements.

\section{A. Statistical validation}

In this part we simulate a simple interferometer composed by two hydrophones with those characteristics:

- frequency: $455 \mathrm{kHz}$

- baseline spacing: $6.5 \lambda$

- baseline tilt-angle: $30^{\circ}$

- across-track aperture: $30^{\circ}$

- along-track aperture: $0.4^{\circ}$

- (square) pulse length: $0.0 \mathrm{~ms}$

- across-track simulation angle: 10 to $80^{\circ}$

The seafloor was simulated using :

- homogeneous depth: $15 \mathrm{~m}$

- homogeneous backscattered strength

In order to estimate the standard deviation of the produced interferometric phase, we simulate several hundred pings over the simulated flat seafloor The principle of the validation is to compare the computed standard deviation to what is predicted by the theoretical formulas presented in [4].
The resulting standard deviation is mainly a result of the combination of three effects

- the baseline decorrelation

- the footprint-shift

- the estimated signal-to-noise-ratio (SNR)

Each theoretical contribution is computed separately and then combined to obtain a global model. This model is compared to what is measured on the generated phase. The result of the comparison is shown in Figure 3.

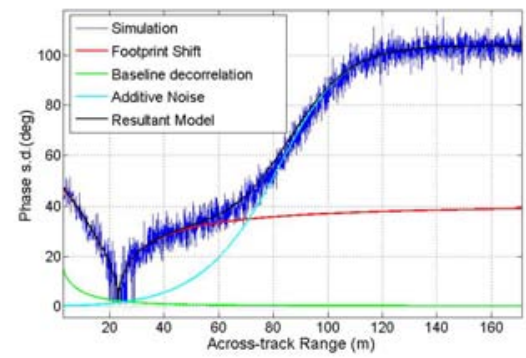

Figure 3. Phase standard deviation $\left(^{\circ}\right)$ depending on the across distance. Light blue: SNR contribution; Red: footprint-shift contribution; Green: baseline decorrelation contribution; Black: resulting model; Dark blue: standard deviation from simulated data

The result shows a very good agreement between the theoretical formulas and the output of our simulated signals

\section{B. Backscattering strength validation}

In order to validate the good behaviour of the backscattered strength of the seafloor, a complete MBES simulation has been realised. We defined a large flat area over which the simulated vessel goes in straight line. The simulated system was given the following characteristics :

- $300 \mathrm{kHz}$ system

- along-track aperture: $1.5^{\circ}$

- across-track aperture: $1.5^{\circ}$

- pulse (square) length: $100 \mathrm{~ms}$

- across-track angle simulation: $120^{\circ}$

The seafloor was described as:

- homogeneous depth: $20 \mathrm{~m}$

- homogeneous backscatter strength (BS): $-30 \mathrm{~dB}$

The simulated sonar surveyed the generated seafloor in straight line for 40 pings to get an averaging of the BS according to the steering angle. In order to compare with real MBES signals the same processing as implemented in the sounders was performed: a time varying gain law was applied on the signal before beamforming, and all necessary compensations were applied on each detected amplitude sample after beamforming.

The necessary compensations are thoroughly described in [5]. This gives a BS measurement similar to what is being done in our current sonar systems. 
With this configuration we obtain the results described in Figure 4.

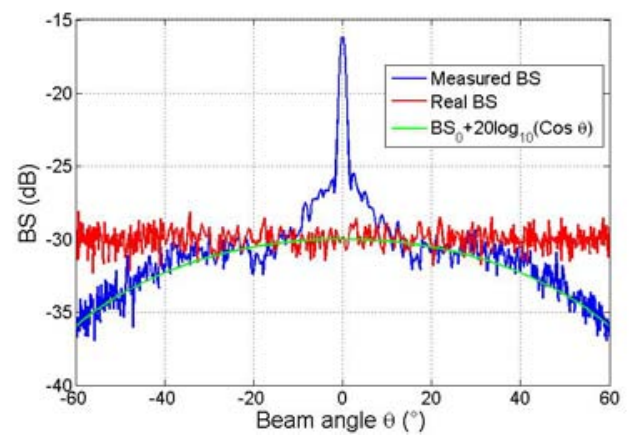

Figure 4. Backscattered strength on detected sample averaged over 40 pings. Blue: measure; Red: reality; Green: curve of $B S_{0}+10 \log _{10}(\theta)$

As shown on the data the simulated measurement results respect Lambert's Law for grazing angles measurement. This was expected since the response from the extended scatterers was made from this model.

Then we observe a transition, from about 5 to $15^{\circ}$ where there is the mixed effect of backscattered and specular signal. At nadir the increase in BS is the effect of mirror reflection. This measurement confirms that the simulation provides a complete and presumably correct description of the signal strength backscattered by a seafloor. Nevertheless, more work needs to be done on the transition regime and on the forward scattered signal simulation part to ensure that it fits with existing theoretical models.

\section{APPliCATION: MBES SURVEy SIMULATION ON A COMPLEX SEAFLOOR}

In this part, the simulation model was used to generate MBES signals acquired over a seafloor containing both large features and smaller sand waves $(20 \mathrm{~cm}$ amplitude and 1 $\mathrm{m}$ period) with an homogeneous $\mathrm{BS}$ equal to $-30 \mathrm{~dB}$. The generated seafloor is presented in Figure 5.

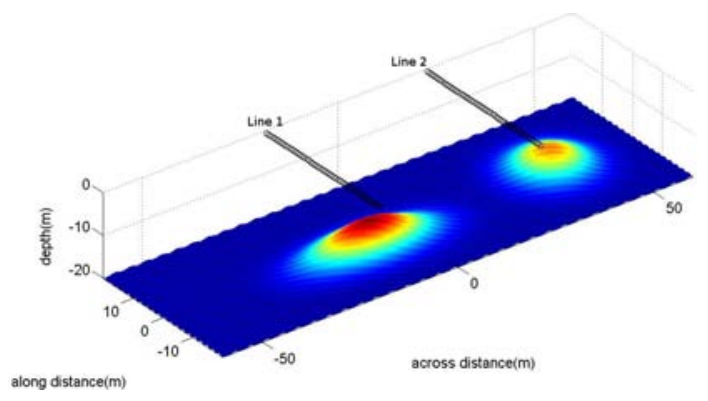

Figure 5. Generated seafloor and antenna positioning during the "survey" (black circles)

The MBES system used has the following characteristics :

- $300 \mathrm{kHz}$ system
- aperture along track: $1.5^{\circ}$

- aperture across track: $1.5^{\circ}$

- pulse (square) length: $300 \mathrm{~ms}$

- across-track angle simulation: $120^{\circ}$

Each ping generates the complex signal for each receiving hydrophone of the antenna. This signal is then beamformed to create a complete water column view in amplitude and interferometric phase. Figure 6 shows one of the generated ping.

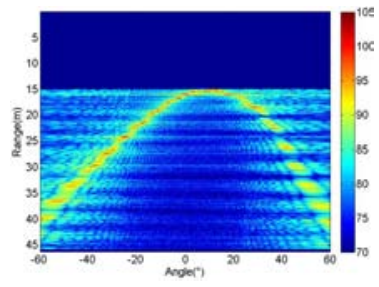

(a) Amplitude measurement ( $d B$ )

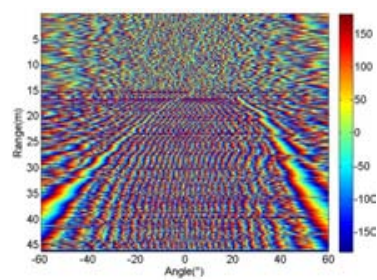

(b) Phase measurement $\left({ }^{\circ}\right)$
Figure 6. Simulated backscattered signal after beamforming as a function of beam angle $\left({ }^{\circ}\right)$ and range $(\mathrm{m})$, in amplitude (left) and phase (right) for one ping

A simple bottom detection algorithm is then applied to each ping, to get the bathymetry from phase and amplitude data. A synthesis from both measurements is done based on the estimated quality of the resulting soundings.

We obtain the bathymetry shown on Figure 7 and the backscatter strength (separated per line) shown on Figure 8.

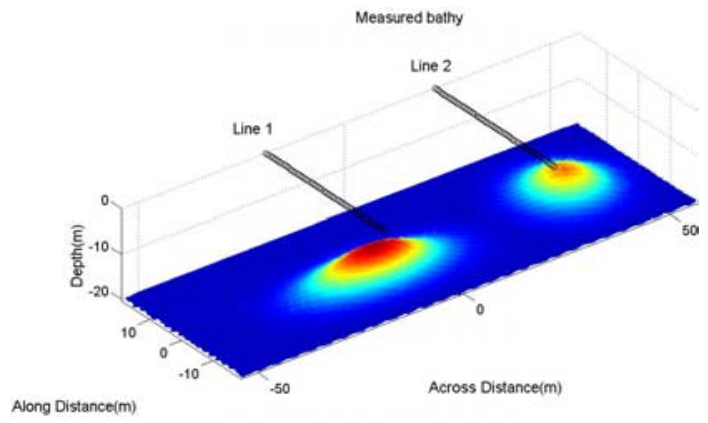

Figure 7. Measured bathymetry and antenna positioning during the "survey" (black circles)

The computed bathymetry provides here a good measurement of the seafloor and restores both macro-relief (the two large mounts) and the sand-waves present on the input model. This allows us to do a preliminary performance evaluation of the simulated system.

Regarding the measured backscattered strength of the seafloor, we observe that it shows the fluctuations of the strength of the acquired signal according to the geometry of the seafloor. 
Again both macro-relief and small waves of the seafloor can be observed on the resulting backscattering strength signal. This confirms that the generated BS behaves correctly.

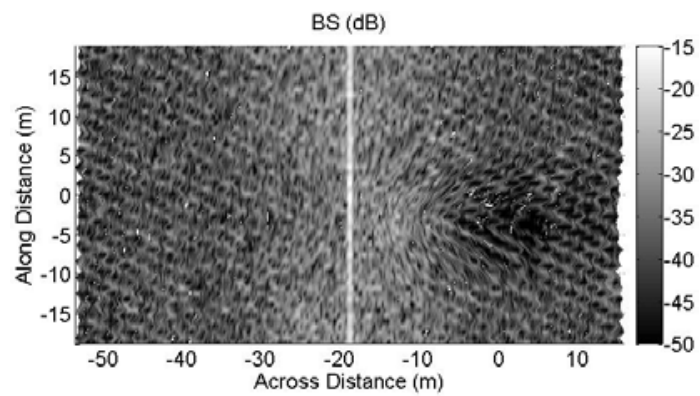

(a) BS line $1(d B)$

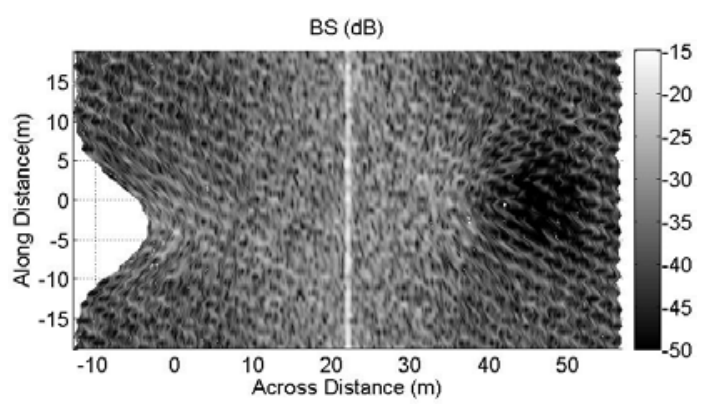

(b) BS line $2(d B)$

Figure 8. Measured backscattered strength (BS) on line 1 (top) and 2 (bottom) corrected according Lambert's Law

Consequently, it seems that the validations done in Part III-A are still valid for a more complex situation. Indeed, the generated signals made it possible to perform a complete beamforming and a relevant BS measurement at the same time, even on a very fluctuating seafloor.

\section{CONCLUSION}

This paper is a first study of the potential of the extended scatterers model in signal simulation. It shows good results associated with the use of a very limited computational power. Indeed, the simulation results presented here were obtained on an ordinary laptop running Matlab. Of course a lot of improvements can be done to the model, such as the introduction of a non constant celerity profile, but this first assessment confirms the many possibilities offered by this modelling. At the moment it does not offer a complete description of the acoustical environment as done in [9], it only demonstrates the simplicity and possible efficiency of the extended scatterers model.

In the future, this simulation could be updated to take more physical phenomena into account, such as multipath, diffraction and Doppler effect. Nevertheless, all those possible development will be undergone keeping in mind that the resulting algorithm has to be able to run with a limited computational power available.

\section{REFERENCES}

[1] S. Daniel, D. Gueriot, and E. P. Maillard, 'Underwater environment restitution through sonar images and swath bathymetry rendering', IEEE Int. Conf. Systems, Man, Cybernetics, vol. 5, Oct. 1998, pp. 4417-4422.

[2] D. Gueriot, C. Sintes, and R. Garello, 'Sonar data simulation based on tube tracing'

[3] D. Gueriot, C. Sintes, 'Forward Looking Sonar Data Simulation through Tube Tracing', OCEANS 2010 IEEE - Sydney, Australia, 24-27 May 2010 in OCEANS 2007- Europe, 18-21 June 2007, pp. 1-6.

[4] X. Lurton, 'Swath bathymetry using phase difference: theoretical analysis of acoustical measurement precision', IEEE J. of Oceanic Eng., 25(3), pp. $351-363,2000$

[5] Lurton X., An Introduction To Underwater Acoustics, Principles and Applications, Second Edition, Springer-Verlag, Berlin, 2010

[6] M. Palmese, A. Trucco, 'A Simulation Method for the Design of a 3-D Acoustical Imaging System for Sub-Bottom Investigation', IMTC 2005, Ottawa, Canada, 17-19 May 2005

[7] C. Sintes, D.Gueriot, 'Interferometric sonar simulation through tube tracing', IEEE OCEANS 2008 - MTS/IEEE Kobe Techno-Ocean, 8-11 April 2008, pp. 1-8

[8] Mitchell, Richard L. Radar Signal Simulation G.E. Pallon and J.F. Walker. Santa Monica: Mark Resources, 1985. Print.

[9] R.P. Goddard, 'The Sonar Simulation Toolset, Release 4.1: Science, Mathematics, and Algorithms,' APL-UW TR 0702, Applied Physics Laboratory, University of Washington, Oct 2008. 